\title{
Event-related potential data acquisition on the Apple II+/IIe
}

\author{
TYLER S. LORIG \\ Augustana College, Rock Island, Illinois
}

\begin{abstract}
Event-related potential (ERP) research is a valuable technique for understanding neural mechanisms underlying cognition. With few exceptions, this research has used minicomputerbased data-acquisition systems, thus limiting ERP research to labs with large computers. The hardware and software presented here allow the Apple II + or Apple Ile microcomputers to present stimuli and collect data from as many as three electrode sites. A fourth electrode site may be used to monitor eyeblinks and allows the exclusion of those trials in which an eyeblink occurs. After the data are collected and averaged, they are stored by subject, task, and electrode on disk where they subsequently may be displayed, analyzed, or downloaded to a mainframe computer.
\end{abstract}

The event-related potential (ERP) has provided psychophysiologists with data valuable to the study of human information processing. Typically, ERP dataacquisition systems have used general purpose laboratory minicomputers such as Digital Equipment Corporation's PDP-11 series (Pavel, 1981). There are few reports of microcomputer-based ERP systems. Criswell and Babcock (1978) report an evoked potential data-acquisition system based on the KIM microcomputer. This system uses an inexpensive single-board microcomputer and relies on external stimulus production (photic stimulator). Because this system uses a simple stimulus and has little capacity to store ERP data, it seems unsuitable for the demands of research on human information processing.

Commercially available systems which use microcomputers also have limited flexibility. Several manufacturers produce equipment designed to capture and average a waveform following a signal from the computer. Following another signal, the external device dumps its contents into the microcomputer for storage. Because this external device is not capable of comparing waves, the microcomputer must process the waveforms one at a time if eye movements are to be excluded or if the stimuli presented are within different categories. This manner of processing reduces the function of this device to a storage buffer for the individual ERP wave, a function which the computer alone is capable of performing.

This paper presents a description of the hardware and software for a microcomputer-based ERP data-acquisition system. This system is flexible in terms of stimulus administration and allows up to five categories of stimuli to be presented to the subject. Following completion of stimulus administration, the data from as many as three electrode sites may be averaged and saved in a disk file.

Development of this program was supported by a grant to the author from the Augustana Faculty Research Committee. The author's address is: Department of Psychology, Augustana College, Rock Island, IL 61201 . Copies of the programs (in DOS 3.3 format) may be obtained by sending a self-addressed, stamped disk mailer and disk to the author.

\section{HARDWARE}

The hardware used in the data acquisition system consists of two inexpensive, commercially available circuit boards and a hand-held response device. The two circuit boards (John Bell Engineering, 400 Oxford Way, Belmont, CA 94002) are a peripheral interface board (\$70) and an analog-to-digital converter (ADC) board (\$90). Both are designed to be mounted in the Apple II motherboard. The software requires the peripheral interface board to be mounted in Slot 4 and the ADC to be mounted in Slot 5.

The peripheral interface board consists of two versatile interface adapters (VIA). Each of these devices contains two 16-bit timers and two 8-bit input/output ports. A simple two-switch device (see Figure 1) is connected to the $\mathrm{J} 3$ port of the interface and provides the manipulanda for assessing subjects' responses to stimuli.

The ADC board is an 8-bit, 16-channel, successive approximation-type converter. It has a $0-$ to $5-\mathrm{V}$ DC, high impedance input range and high linearity. The board also offers a relatively fast conversion time $(100 \mu \mathrm{sec}$ per chan-

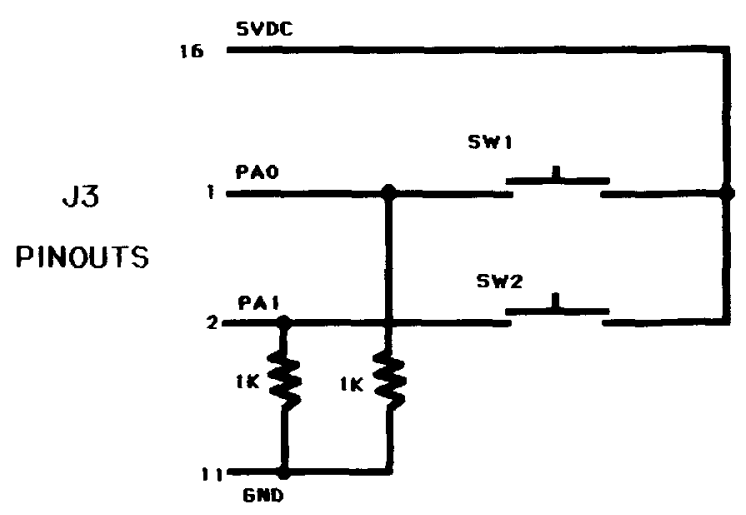

Figure 1. Schematic diagram of the two-pushbutton response device. 
nel). The choice of an 8-bit ADC rather than an ADC with greater resolution is based on several factors. Because the Apple contains an 8-bit microprocessor and 8bit data bus, interface and software for the ADC is less expensive and less complicated. The more complicated software necessary to convert and store the ERP would produce greater time demands in critical subroutines and would lead to a lower sampling rate or a decrease in the number of channels digitized. Use of an 8-bit ADC has been criticized because it divides the range of the ADC ( 0 - to 5-V DC in this case) into 256 subunits. Also, because the entire range of the ADC generally is not used, the number of subdivisions is somewhat less than 256 . For instance, a $50-\mu \mathrm{V}$ (peak-to-peak) signal might occupy a range of $200 \mathrm{ADC}$ subdivisions, which means that each microvolt would be represented by only four divisions; therefore, an 8-bit converter would be unable to discriminate changes in the signal of less than $.25 \mu \mathrm{V}$. Tenand 12-bit converters provide greater discrimination.

The ERP data acquisition system is dependent on the computer, ADC, and amplifier system. The accuracy of the amplifiers, which provide the input signal, also must be considered in the selection of the ADC. Nonlinearity and especially amplifier noise may degrade system performance. For instance, even high quality EEG amplifiers such as the Colburn Instruments S75-03 (High Performance Bioamplifier) have typical noise specifications (within the EEG frequency range) of $.12 \mu \mathrm{V}$ with a maximum of $.35 \mu \mathrm{V}$. With noise levels in this range, efforts to discriminate fluctuations of less than $0.25 \mu \mathrm{V}$ would be wasted. Given these limitations, the resolution of the input signal attainable with an 8-bit ADC is likely to be sufficient for most applications.

Interface of the ADC to external amplification equipment is described in the literature provided by the manufacturer. Some investigators may wish to optically isolate this interface to ensure subject safety because connection with the Apple power supply may compromise the medical isolation of the amplifying unit. The exact nature of this interface depends on the investigator's amplifiers.

\section{SOFTWARE}

The software for ERP data acquisition consists of three BASIC programs linked by a menu server. The menu program is also the HELLO program so that it "boots" automatically when the Apple is turned on. The listing for this program is presented in Table 1 . The menu allows the selection of one of three programs: CREATE, ERP DATA, and GRAPH ERP.

The CREATE program produces a stimulus file for administration to the subject. The program is interactive and asks the user for the number of items which will be placed in the file. The user is prompted next to enter the stimulus item, the category of the stimulus item, and the coding for the correct response. Categories may range from one to five and represent one task or independent varia-
Table 1

Listing for File HELLO

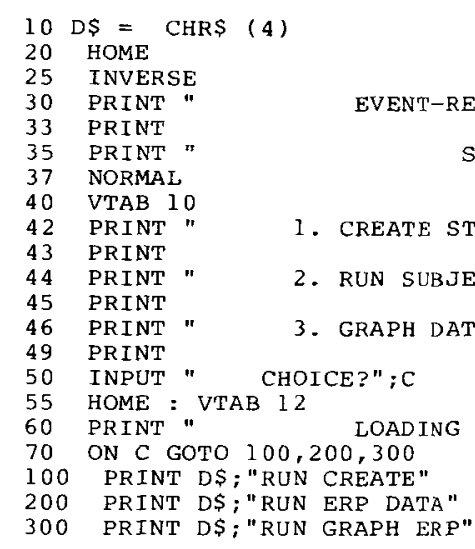

ble in the data collection. In a list of consonant/vowel/consonants (CVCs), for instance, the investigator may specify nonsense syllables to be in one category and words in another. Responses are coded by the pushbutton designated as the correct choice. In the example above, the subject may be instructed to respond on pushbutton " 1 " if he/she recognizes the stimulus as a word and to respond on pushbutton ' 2 "' if the stimulus is not a word. Two items which might appear in this stimulus list would be entered as follows: CAT,2,1 and TAF,1,2.

When the specified number of stimuli have been entered, the user is prompted to enter a filename for storage of the stimuli. After the filename is specified, the items are stored, and the user is returned to the main menu. The listing for CREATE is provided in Table 2.

The ERP DATA program (see Table 3 ) is the datacollection program in the set and uses two machine language programs, which are loaded during execution of the main ERP DATA program. Program flow for data collection is relatively simple. First a buffer area is established at the top of working memory to protect the machine language programs and the data collected after each stimulus from being overwritten by program variables. The two machine language programs are loaded into the buffer area.

The user is then prompted to enter the subject number, the name of the file containing the stimulus items (Task file), the number of items in this file, the number of categories of stimulus items, the number of cortical electrodes in use (not counting the EOG electrode), and the eyeblink correlation cutoff. The stimulus file and eyeblink template (BLINK) are read into memory, and the first stimulus is selected. A fixation point appears on the screen followed by the stimulus item. The computer then executes the machine language subroutine called ERP (Table 4). ERP sets up a timed data-collection loop using the VIA timers so that a sample is collected from each electrode at 4-msec intervals for a total of 256 samples for each electrode. Each sample is stored sequentially in the 
Table 2

Listing for File CREATE

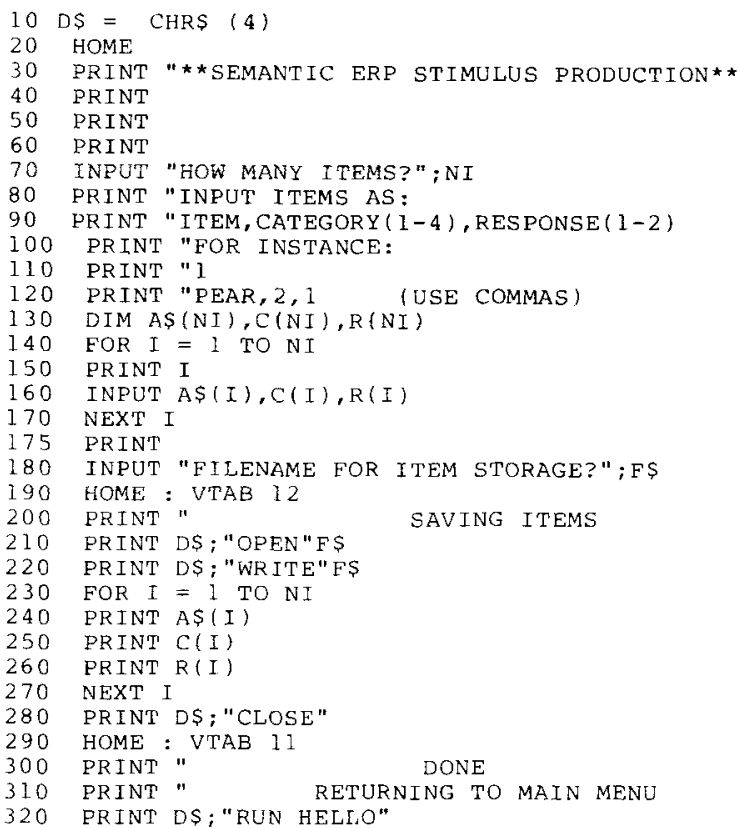

buffer area mentioned earlier. Samples are collected by executing a nested subroutine called $A / D$. This program is outlined in literature provided by the manufacturer. A modified version is provided in Table 5 .

After the 256 samples have been collected, program flow returns to the main BASIC program where the screen is cleared, and after a short delay, the subject is instructed to respond. The program scans the response device until a response is made on one of the pushbutton switches. When the response is made, the program checks to see if it is correct for the stimulus item presented. Following this, a correlation coefficient is obtained for the blink template and the EOG channel. If the correlation coefficient is less than that specified at the beginning of the program and the response was correct, the trial is considered valid and the data in the electrode buffers are added to indexed arrays. The number of valid trials for the category is also incremented. Following this determination, the next stimulus item is presented and the same routines are executed until all of the stimuli have been presented. After stimulus presentation is complete, the subject is informed and instructed to relax. At this point, the data are averaged in a simple summative fashion by dividing the total for each electrode, category (task), and time bin by the number of trials which were found to be valid for that category. The user is then instructed to insert a disk for data storage and press the return key. After doing this, the data are saved by subject number, task, and electrode number. Each data file is labeled as SxTXEx and contains the 256 data points corresponding to the 4-msec time bins. Also displayed are task (category) numbers and the number of valid trials collected for that task. The program disk should be reinserted, and the user may respond to the "RETURN TO MAIN MENU" question. An answer of " $Y$ " results in reexecution of the menu program. The program listing for ERP DATA is presented in Table 3.

The GRAPH ERP program (see Table 6) provides a means to display the previously obtained ERP waveforms in the Apple's high resolution graphics mode. The user may choose to display a particular file, clear the screen, or exit the program and return to the main menu. To use this program the operator should select alternative " 3 "' from the main menu. The GRAPH ERP program will load and begin execution. Instructions regarding the commands are listed, and then the display is switched into the high resolution mode. To select a file for view, the "WHICH?" question is answered with " $F$ ". The user then is asked for the filename of the data to be displayed. After answering this question, the data file is graphed. If an overlay of another ERP is desired for comparison purposes, another file is specified for display. The screen may be cleared with the " $\mathrm{C}$ " command, and the user may exit to the main menu with the " $E$ " command. The images on the screen may be printed using commercially available screen "dump" utilities. To use one of these utilities, it is necessary to escape from the program by typing $~ C \mathrm{C}$ and then run the utility.

Table 3

Listing for File ERP DATA

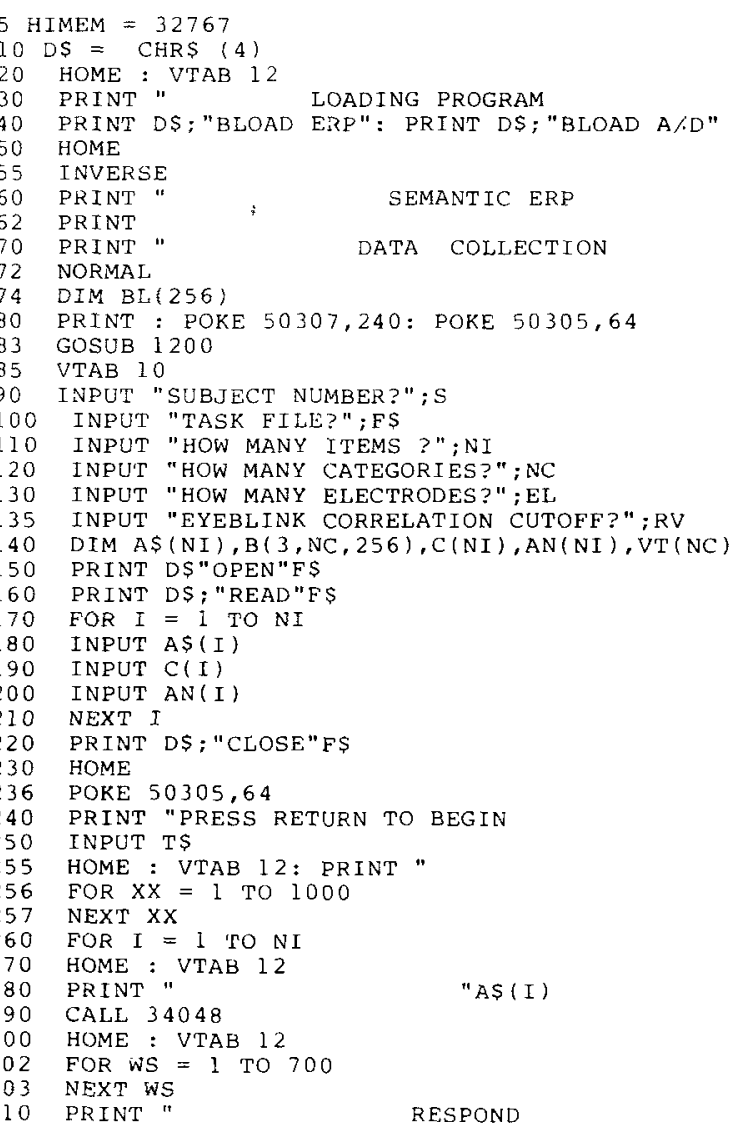


$320 \mathrm{KD}=\operatorname{PEEK}(50305)-76$

330 IF $K D=1$ OR $K D=2$ THEN GOTO 340

332 GO'TO 320

340 HOME : VTAB 12

350 PRINT "

360 GOSUB 2000

370 IF BR > = RV THEN GOTO 1000

$380 \mathrm{TC}=\mathrm{C}(\mathrm{I})$

$390 \mathrm{TZ}=\mathrm{AN}(\mathrm{I})$

400 IF KD<> TZ THEN GOTO 1000

$410 \mathrm{VT}(\mathrm{TC})=\mathrm{VT}(\mathrm{TC})+1$

420 FOR $\mathrm{E}=0 \mathrm{TO} 255$

$430 \mathrm{~B}(1, \mathrm{TC}, \mathrm{E})=\mathrm{B}(1, \mathrm{TC}, \mathrm{E})+\operatorname{PEEK}(34304+\mathrm{E})$

$440 \mathrm{~B}(2, \mathrm{TC}, \mathrm{E})=\mathrm{B}(2, \mathrm{TC}, \mathrm{E})+\operatorname{PEEK}(34560+\mathrm{P})$

$450 \mathrm{~B}(3, \mathrm{TC}, \mathrm{E})=\mathrm{B}(3, \mathrm{TC}, \mathrm{E})+\operatorname{PEEK}(34816+\mathrm{E})$

470 NEXT E

480 NEXT I

490 HOME : VTAB 1

500 PRINT" " TASK COMPLETED

510 PRINT"

520 FOR $E=1$ TO ET

530 FOR $T=1$ TO NC

540 FOR $I=0$ TO 255

$550 B(E, T, I)=B(E, T, I) / V T(T)$

560 NEXT I

570 NEXT T

580 NEX'T E

590 PRINT "SAVING DATA"

600 PRINT "INSERT DATA DISK"

610 PRINT "PRESS RETURN TO BEGIN

620 INPUT QS

630 FOR $\mathrm{E}=1$ TO EL

640 FOR $\mathrm{T}=1$ TO NC

650 PRINT DS: "OPEN S"S "T "T "E"E

660 PRINT DS: "WRITE S"S"T"T"E"E

670 FOR I $=0$ TO 255

680 PRINT $B(E, T, I)$

690 NEXT I

700 PRINT D\$; "CLOSE"

710 NEXT T

720 NEXT E

730 HOME

740 PRINT "DATA SAVED"

750 PRINT "RECORDED RESPONSES"

760 FOR $\mathrm{T}=1$ TO $\mathrm{NC}$

770 PRINT $\mathrm{T}, \mathrm{VT}(\mathrm{T})$

780 NEXT $T$

790 PRINT

800 PRINT

810 PRINT" * * END OF PROGRAM * * "

830 INPUT "MENU?";Q\$

840 IF $Q \$=$ "Y" THEN GOTO 860

860 PRINT DS; "RUN HELLO"

870 END

1000 FOR $X X=1$ TO 4500

1100 NEXT $X X$

1110 GOTO 480

1200 PRINT DS: "OPEN BLINK"

1210 PRINT D\$; "READ BLINK"

1220 FOR $Q X=1$ TO 256

1230 INPUT BL(QX)

1240 NEXT QX

1250 PRINT DS; "CLOSE"

1260 RETURN

2000 FOR QX $=1$ TO 256 STEP 8

$2010 \mathrm{~K}=\operatorname{PEEK}(35071+\mathrm{QX})$

$2020 \mathrm{~F}=\mathrm{BL}(\mathrm{OX})$

$2030 \mathrm{Kl}=\mathrm{Kl}+\mathrm{K}: \mathrm{Fl}=\mathrm{Fl}+\mathrm{F}$

$2040 \mathrm{~K} 2=\mathrm{K} 2+\mathrm{K} * \mathrm{~K}: \mathrm{F} 2=\mathrm{F} 2+\mathrm{F} * \mathrm{~F}$

$2050 \mathrm{H}=\mathrm{K} * \mathrm{~F}$

$2060 \mathrm{HS}=\mathrm{HS}+\mathrm{H}$

2070 NEXT QX

$2080 \mathrm{Rl}=((32 * \mathrm{HS})-(\mathrm{Kl} * \mathrm{Fl}))$

$2090 \mathrm{R} 2=\operatorname{SQR}(((32 \star \mathrm{K} 2)-(\mathrm{K} 1 * \mathrm{~K} 1)) \star$

$((32 * F 2)-(F 1 * F 1)))$

$2100 \mathrm{BR}=\mathrm{R} 1 / \mathrm{R} 2$

$2110 \mathrm{~K} 1=0: \mathrm{K} 2=0: \mathrm{Fl}=0: \mathrm{F} 2=0: \mathrm{HS}=0$

2120 RETURN

Table 4

Listing for File ERP

SOURCE FILE - ERP

$\begin{array}{llllll}0 & & & & ; & \\ 1 & & & & \text { ORG } \$ 8500 \\ 2 & 8500 & \text { A9 } & 00 & & \text { LDA } \$ \$ 00\end{array}$

\begin{tabular}{|c|c|c|c|c|c|c|}
\hline 8502 & $8 \mathrm{D}$ & 81 & $\mathrm{C} 4$ & & STA & $\$ C 481$ \\
\hline 8505 & A2 & 00 & & & LDX & $\# \$ 00$ \\
\hline 8507 & A9 & 90 & & AGAIN & LDA & $\# \$ 90$ \\
\hline 8509 & $8 D$ & 88 & C4 & & STA & $\$ C 488$ \\
\hline $850 \mathrm{C}$ & A9 & $0 F$ & & & LDA & $\#$ \# $0 \mathrm{~F}$ \\
\hline $850 \mathrm{E}$ & $8 D$ & 89 & $C 4$ & & STA & \$C489 \\
\hline 8511 & $A D$ & $8 \mathrm{D}$ & $\mathrm{C} 4$ & LOOP & $\mathrm{LDA}$ & $\$ C 48 D$ \\
\hline 8514 & 29 & 20 & & & AND & $\# \$ 20$ \\
\hline 8516 & Fo & F9 & & & $\mathrm{BEQ}$ & LOOP \\
\hline 8518 & 20 & 60 & 82 & & JSR & $\$ 8260$ \\
\hline $851 \mathrm{~B}$ & $A D$ & AO & 82 & & LDA & $\$ 82 A 0$ \\
\hline $851 \mathrm{E}$ & 90 & 00 & 86 & & STA & $\$ 8600, x$ \\
\hline 8521 & $A D$ & 61 & 82 & & LDA & $\$ 82 \mathrm{Al}$ \\
\hline 8524 & 9D & 00 & 87 & & STA & $\$ 8700, x$ \\
\hline 8527 & $A D$ & 62 & 82 & & LDA & $\$ 82 \mathrm{~A} 2$ \\
\hline $852 \mathrm{~A}$ & 9D & 00 & 88 & & STA & $\$ 8800, x$ \\
\hline $852 \mathrm{D}$ & $A D$ & 63 & 82 & & LDA & $\$ 82 A 3$ \\
\hline 8530 & $9 D$ & 00 & 89 & & STA & $\$ 8900, x$ \\
\hline 8533 & E8 & & & & INX & \\
\hline 8534 & EO & 00 & & & CPX & $\# \$ 00$ \\
\hline 8536 & Do & $\mathrm{CF}$ & & & BNE & AGAIN \\
\hline 8538 & A9 & 40 & & & LDA & $\# \$ 40$ \\
\hline $853 \mathrm{~A}$ & $8 \mathrm{D}$ & 81 & C4 & & STA & $\$ C 481$ \\
\hline & 60 & & & & RTS & \\
\hline
\end{tabular}

Table 5

Listing for File $A / D$

SOURCE FILE - A/D

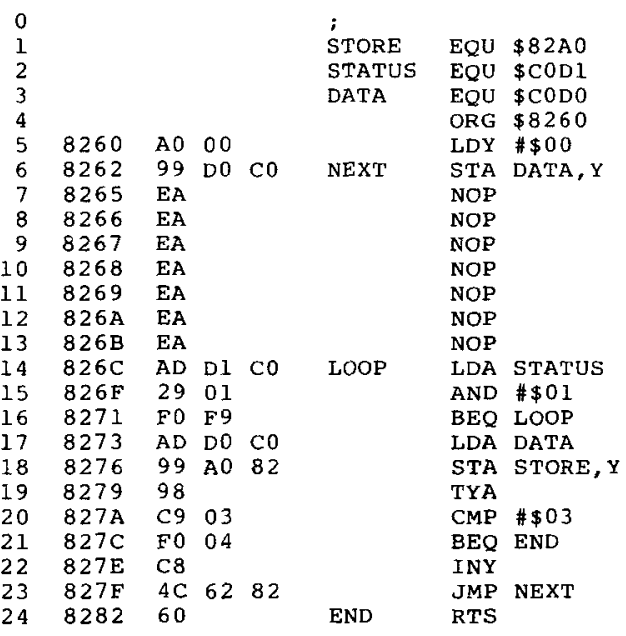

Listing for File GRAPH ERP

$10 \mathrm{DS}=$ CHRS $(4)$

20 HOME

26 VTAB 12

30 PRINT "ENTER ' $F$ ' FOR FILE FOR DISPLAY"

35 PRINT " 'C' FOR CLEAR"

40 PRINT" ' $\mathrm{E}$ ' FOR END"

45 FOR $I=1$ TO 2000

50 NEXT I

55 DIM A (256): VTAB 24

60 HGR

$70 \mathrm{HCOLOR}=7$

100 INPUT "WHICH $(\mathrm{F}, \mathrm{C}, \mathrm{E})$ ? ", OS

110 IF OS $=$ "F" THEN GOTO 500

120 IF $Q S=$ "C" THEN GOTO 600

130 IF Q\$ $=$ "E" THEN GOTO 700

140 GOTO 100

500 INPUT "FILENAME?" ; F

505 INPUT "GAIN?"; GA

$\begin{array}{ll}505 & \text { INPUT "GAIN?"; GA } \\ 510 & \text { PRINT D\$"OPEN"F\$ }\end{array}$

$\begin{array}{lll}510 & \text { PRINT DS"OPEN"F\$ } \\ 520 & \text { PRINT D\$"READ"F\$ }\end{array}$

$530 \mathrm{FOR} I=1$ TO 256

540 INPUT A(I)

550 NEXT I 


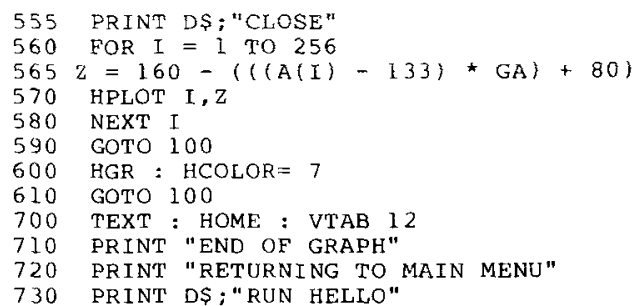

\section{SYSTEM IMPLEMENTATION}

In order for the program to operate correctly, all files (HELLO, ERP DATA, CREATE, GRAPH ERP, A/D, ERP, BLINK, and the file with the stimulus items) must be on the same disk. After typing and saving the program files, it is an easy matter to create a test item file using the "CREATE A STIMULUS FILE" alternative from the main menu. Creating the BLINK file is somewhat more difficult. The collection program must have a file called BLINK to use in establishing the EOG correlation. To create a usable BLINK file, the amplifier associated with ADC channel 0 is used to amplify EOG. Lines 83 and 360 must be deleted from the ERP DATA program; then the data collection routine is run and subject number 0 is specified. Then the task file and parameters are selected in the stimulus file. The use of only one item from the task file must be specified. The number of electrodes should be 1 and the correlation cutoff should be greater than 1. The program continues, and the subject is allowed to respond to the single trial. The data are stored and the subject's file (SOT1E1) is examined to determine whether an eye blink occurred in response to the stimulus. If no blink occurred, the procedures outlined above are repeated (the deletions of lines 83 and 360 will be necessary each time ERP DATA is run from the menu). If an eye blink did occur, the data file SOT1E1 is renamed to BLINK.

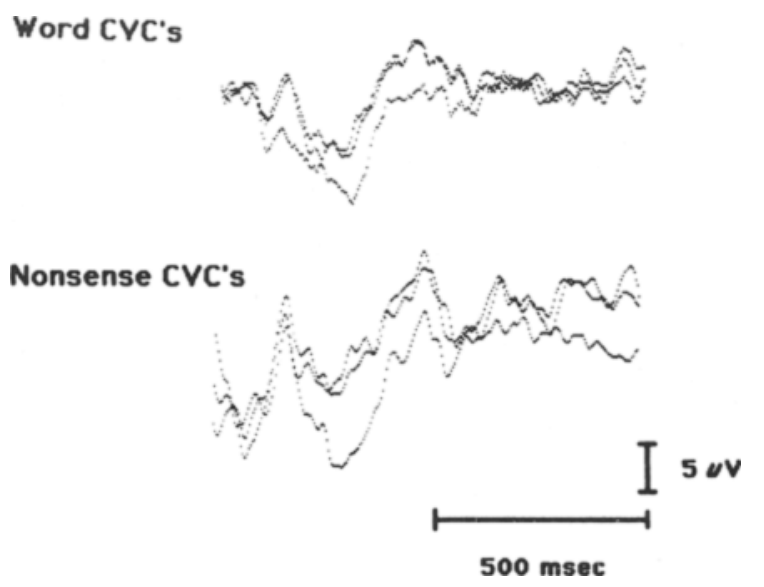

Figure 2. ERP data collected from three electrode sites on the CVC word and nonsense syllable task (negative is up).
The ERP DATA program may now be run from the menu with no changes; ADC channels 0,1 , and 2 may be used for cortical recording; and ADC channel 3 may be used for EOG.

Stimulus presentation is accomplished by the Apple II monitor. In order to facilitate presentation, a second remote video monitor may be added by the use of an RCA phono " $Y$ " adapter and cable connected to the video output port on the Apple. This allows the subject and the operator to see the computer screen without interacting during stimulus presentation.

\section{DEMONSTRATION}

To demonstrate the use of the program, a single subject was tested using CVC nonsense syllables and CVC words. The subject was instructed to respond to words by pressing pushbutton " 1 " and to nonsense syllables by pressing pushbutton " 2 ." Data were collected from three electrode sites all referenced to linked mastoids $(\mathrm{Fz}, \mathrm{Cz}$, and $\mathrm{Pz}$ ). EEG was amplified by a Beckman Accutrace $200 \mathrm{~A}$ electroencephalograph with gain set so that a 50$\mu \mathrm{V}$ (peak-to-peak) potential produced an ADC range of 190 units. The eyeblink correlation cutoff was set to 7 . The data collected from this subject were graphed and then printed. They are presented in Figure 2.

\section{LIMITATIONS}

While this ERP data collection system offers unique advantages to some users, there are also limitations, the most serious of which is the rather lengthy interstimulus interval (ISI). Because the program must calculate a correlation coefficient and then add three sets of 256 numbers to indexed arrays during the ISI, a 22-sec delay results. Only one EOG electrode is used because calculation of two correlation coefficients would considerably increase the ISI. A version of the data collection program compiled with Microsoft's TASC compiler produces an ISI of $6 \mathrm{sec}$ and greatly increases the usefulness of the program. Because this system uses an 8-bit ADC, the operator must pay close attention to dynamic range and resolution of the input signal.

Even with these limitations, this system offers the user the ability to collect reliable ERP data with a low cost microcomputer-based system.

\section{REFERENCES}

CrIswell, H. E., \& BABCock, B. (1978). A flexible low cost signal averager. Electroencephalography \& Clinical Neurophysiology, 45 , 535-537.

PAVEL, M. (1981). Results of a preliminary survey of computer users in psychology laboratories. Behavior Research Methods \& Instrumentation, 13, 272-275.

(Manuscript received March 12, 1985; revision accepted for publication July 16, 1985.) 